\section{Mechanical deductive techniques: Three microcomputer packages}

\section{LAURENCE GOLDSTEIN and TIM MOORE Hong Kong University, Hong Kong}

Cognitive strategies for problem solving are poorly understood. In the case of certain kinds of inference, various algorithms in formal logic furnish a mechanical check of validity, but it is by no means clear that the strategy involved in arriving at an intuitive assessment of the validity of an argument utilizes such formal rules, or proceeds instead by analogy with other arguments or by some alternative means. However, since at fairly low levels of complexity of argument, subjects differ in their intuitive judgments of validity and invalidity, mechanical methods become invaluable. Furthermore, since the techniques are formal, they are applicable to a range of structurally similar problems, for example, to the design of electrical and electronic circuitry. The programs described here teach two kinds of elementary deductive techniques for establishing whether a given conclusion follows logically from a given set of premises. This is of obvious practical value. But whether the acquisition of such effective methods for "getting the right answer" fosters improved habits of intuitive reasoning is a question that is currently under research. In comparison, the availability of pocket calculators to young children does nothing to improve-and may impairtheir habits of intuitive arithmetic calculation.

\section{Programs}

A course of three programs has been developed. The programs are written in APPLESOFT BASIC.

JOHN. This program introduces the Venndiagrammatic technique for evaluating syllogistic arguments. It includes some tests on the nature of syllogisms and instructs in the method of examining syllogisms for validity by means of Venn diagrams. The program culminates in a test of the student's mastery of the method. Syllogisms are generated by an algorithm that employs (pseudo)random choice of terms and argument form. The student has to determine whether each argument so generated is valid or invalid. He can check his work by calling up the correct Venn diagram, with representations of each premise being shown at user-controlled intervals and accompanied by an appropriate explana-

The authors' mailing address is: Department of Philosophy, Hong Kong University, Pokfulam Road, Hong Kong. tion. The student may choose the number of questions constituting the test, and the program tells the student his or her score and invites the student to take a further test should he or she wish.

LUDWIG. This program teaches techniques for evaluating arguments in propositional calculus. It introduces the student to the language of propositional calculus and teaches the truth tables for each of the logical connectives. A gamelike section is included, in which the student has to find the correct truth value to complete a truth table, and then place it in position by means of the paddles. The program teaches how to diagnose the form of arguments in ordinary language and how to determine the validity or invalidity of arguments by both the long truthtable method and the short "reductio" technique. There is a test on well formedness, and the program culminates in a test in which arguments are generated by an algorithm employing (pseudo)random choice of variables, connectives, and length of formula. Again, for each argument so generated, the student may check his or her evaluation of its validity against the correct answer supplied by the program. The difficulty level of the examples generated varies with the student's performance and is displayed on the screen. At the end of the test, the student's score is revealed and he or she is given the opportunity of another test.

TIARA. This program provides assistance in diagnosing the logical forms of arguments in ordinary English. It is divided into five sections: (1) general introduction, (2) help in recognizing the devices in English that indicate the structure of an argument, (3) help in recognizing the logical structure of a proposition, (4) tests on argument structure, and (5) tests on propositional structure.

There are a great many ways in which the words of a text provide clues to the presence of an argument and to its structure. The program provides assistance in recognizing such clues and in avoiding false leads. Examples are given in which ordinary sentences are analyzed, step by step, with an accompanying commentary. In the test sections, the user is required to perform such analyses, and the program is ready with advice if it is required. An instructor can readily modify the advice and change and expand the examples given.

The length of time needed to complete JOHN varies, in our experience, from about .5 to $1.25 \mathrm{~h}$. LUDWIG takes about the same length of time, but TIARA is a longer program and users sometimes need two sittings. The program is designed in such a way that it is convenient to break off and return for more. In JOHN and LUDWIG, results of users' test scores are written to files on disk. 


\section{System Requirements}

JOHN and LUDWIG require an Apple II with Autostart ROM, APPLESOFT in ROM, 48K RAM, one disk drive, and a color monitor (the programs lose only vivacity, not substance, if a monochrome screen is used). LUDWIG also requires the game paddles or joystick. TIARA, in its present form, has more specialized requirements: a monochrome, as well as a color, monitor, a Data Advanced Products, Inc., light pen for one section of the lesson, and an M \& R Enterprises Sup'r'terminal 80-column card (providing for user-defined characters), although the program may be modified for use with other 80column terminal boards. For those who do not possess one, the light pen, which introduces only an inessential fun element into the program, can be easily written out.

\section{Availability}

The programs are available on floppy diskettes and are accompanied by notes for students and an information sheet for teachers. It is requested that publications resulting from this software acknowledge this source. Further details may be obtained from: The Secretary, Department of Philosophy, Hong Kong University, Pokfulam Road, Hong Kong.

\section{REFERENCE}

Goldstein, L., \& Moone, F. C. T. Meet MICAL-Teaching elementary logic by microcomputer. Australian Logic Teachers Journal, 1982, 6, 15-29.

(Revision accepted for publication August 16, 1983.) 